\title{
Continuation of auditory frequency gradients across temporal breaks: The auditory Poggendorff
}

\author{
IRWIN POLLACK \\ Mental Health Research Institute, The University of Michigan, Ann Arbor, Michigan 41809
}

\begin{abstract}
An auditory analog of the visual Poggendorff figure was constructed on the assumption that selected temporal variables in audition have parallels with selected spatial variables in vision. As with the visual Poggendorff, the extent of underestimation within the auditory analog is directly related to the slope of the signal and to the magnitude of the temporal gap being bridged. Some of the conditions which limit the existence region of the auditory Poggendorff are determined.
\end{abstract}

Among the many visual illusions, the Poggendorff has been among the most extensively examined in recent years, perhaps because its component parts lend themselves to independent manipulation and analysis (e.g., see Weintraub \& Krantz, 1971). The present study examines a possible auditory analog of the Poggendorff illusion.

The rationale follows that of Julesz and Hirsh (1972). The temporal extent in audition is considered to be the appropriate parallel of the spatial extent in vision. The temporal rate of change in audition, auditory frequency, is considered to be the appropriate parallel of the spatial rate of change in vision, spatial slope. The parallel has been suggested by many theorists, but most vigorously by S. S. Stevens (1975, for a summary of early writings) who argued that a change in auditory pitch is represented by a spatial change in the locus of maximum stimulation of the cochlear partition. By contrast, for example, auditory loudness is represented by an increment in activity at the same location of the cochlear partition.

The specific question asked was how well can a listener extrapolate an auditory temporal gradient through a temporal gap, and whether such behavior follows the major findings with the visual Poggendorff. The specific question may also be considered within the more general context of seeking appropriate multidimensional, rather than onedimensional, intersensory comparisons.

\section{METHOD}

\footnotetext{
Approach

The approach is schematically shown in Figure 1. Figure $1 \mathrm{~A}$

The research was supported in part by the National Science Foundation. Ms. Linda Matich supervised the experimental tests; Louis Wojnaroski wrote the experimental and simulation programs.
}

is a representation of a visual Poggendorff. The left and right legs represent spatial gradients. The task of the observer is to align one of the legs for good continuation across the spatial gap. The slope of the spatial gradient is positive in Figure 1A and is negative in Figure $1 \mathrm{C}$. The same schematic may be employed to represent auditory analogs of the Poggendorff. The abscissa represents successive events in time. The ordinate represents auditory pitch, or, more accurately, the time interval between successive auditory pulses. In time, Figure $1 \mathrm{~A}$ represents an auditory signal with an increasing interval gradient, interrupted by a silent break, and then continued after the break. Figure 1C represents an auditory signal with a negative interval gradient. Since pitch is inversely related to the interpulse interval, the pitch gradient will be termed negative in Figure $1 \mathrm{~A}$ and positive in Figure $1 \mathrm{C}$. Most of the present tests employed a positive interval

VISUAL
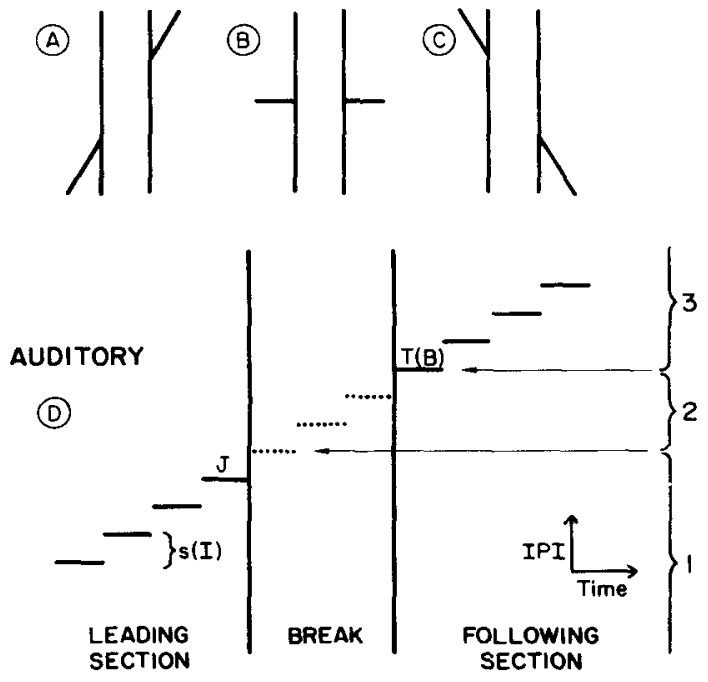

Figure 1. Schematic representation of the visual Poggendorff illusion with a positive slope (A), with a negative slope $(C)$, and the absence of the illusion with a zero slope (B). Panel $D$ illustrates the auditory Poggendorff in terms of a sequence of short-duration tones, separated by a temporal break. The auditory Poggendorff substitutes frequency and time for position and space in the visual Poggendorff. See text and Table 1 for definition of terms. 
Table 1

Notation in Connection with Figure 10

step - a sequence of successive pulses, separated by a constant interpulse interval

IPI - interpulse interval, the duration between two successive pulses

$\mathbf{s}(D)$ - the duration of each step

s(I) - the change in interpulse interval between successive steps

J - the interpulse interval on the last step of the leading section before a silent break

$s(n)$ - the number of steps before and after a silent break; also written as $\mathbf{n}(\mathbf{s})$

$B(D)$ - the duration of the break

M - (not shown) the matching interpulse interval, adjusted by the listener; the listener adjusts the interpulse interval of the initial step of the following section

$T(B)$ - the targeted interpulse interval for matching, assuming accurate extrapolation across the break

$T(\bar{B})$ - the targeted interpulse interval for matching, assuming no break, shown as the left dotted interval of Figure 1

gradient, or a negative pitch gradient. As with the visual Poggendorff, we can independently modify the slopes of the gradient of the leading and of the following sections. We can also modify the extent of the break.

\section{Notation}

In the present tests, the slope was varied in terms of a series of step changes, as illustrated in Figure 1D and listed in Table 1. Time runs along the horizontal scale; interpulse interval runs along the vertical scale. Each "step" consists of a sequence of successive interpulse intervals. An exploded view of each step would reveal a series of pulses. Within any one step, the interpulse interval, IPI, is held constant over the duration of the step, $s(D)$. The differences in the interpulse interval between successive steps is $\mathbf{s}(\mathrm{I})$. The interpulse interval of the step before the temporal break is marked $\mathrm{J}$. The targeted interpulse interval, assuming continuity of successive steps over the break, is $T(B)$. The targeted interpulse interval, assuming no break, or a break of $0 \mathrm{sec}$, is $\mathrm{T}(\overline{\mathrm{B}})$ and is shown as the first dotted line. The section before the break is termed the "leading" section, and, the section after the break is termed the "following" section.

If a listener is asked to adjust the interpulse interval of the first step of following section, his adjustment matches fall into three possible regions. Region 1 is the locus of matches which fail to extrapolate the step gradient beyond the leading section, assuming there is no break. Region 3 is the locus of matches which overextrapolate the step gradient beyond that required for continuity within the break. Region 2 is the locus of matches which underextrapolate the step gradient.

A concrete example might illustrate the sequences. Assume that the initial IPI is $10 \mathrm{msec}$, that the increment between successive steps is $1 \mathrm{msec}$, that two intervals comprise a step, that four steps comprise a section, and that the break is $90 \mathrm{msec}$. With commas denoting successive intervals, semicolons denoting successive steps, and colons denoting successive sections, the sequence of interpulse intervals is: 10,$10 ; 11,11 ; 12,12 ; 13,13: 14,14 ; 15,15$; $16,16: 17,17 ; 18,18 ; 19,19 ; 20,20 \mathrm{msec}$. In terms of this sequence, $\mathrm{J}=13 \mathrm{msec}, \mathrm{T}(\bar{B}) \Rightarrow 14 \mathrm{msec}, \mathrm{T}(\mathrm{B})=17 \mathrm{msec}$. Therefore, in this example, matching adjustments, $M$, of the first interpulse interval of the following section which are greater than $17 \mathrm{msec}$ will be said to represent overextrapolations; matchings below $14 \mathrm{msec}$ will be said to represent failures to extrapolate; matchings greater than $14 \mathrm{msec}$ but less than $17 \mathrm{msec}$ will be said to represent underextrapolations.
Unless specified otherwise, the step duration, $s(D)=125 \mathrm{msec}$, the number of steps, $n(s)=8$; the targeted interval, $T(B)=$ $4 \mathrm{msec}$; and the interval change per step, s(I), was identical for the leading and following sections.

\section{Procedure}

The listener was instructed to adjust the initial interpulse interval of the following section so that the pitch transition from the initial section was as smooth as possible. All other parameters were fixed. A wide range of initial interpulse intervals of the following section, above and below $T(B)$, was employed on separate trials. The listener adjusted $M$ by pressing one button to increase the pitch, and by pressing another button to decrease the pitch. The initial change in $M$ was about $13 \%$ of $T(B)$. Successive changes in direction reduced the change in $M$ by onehalf. Listeners were encouraged to bracket their answers by systematically overshooting and undershooting their final adjustment. In this way, initial changes surveyed a broad pitch landscape and finer and finer adjustments were made as the final match was approached. The smallest unit step change was $1 \mu \mathrm{sec}$. When satisfied with the adjustment, the listener pressed another button which recorded his adjustment. The entire procedure was controlled by a PDP-9 computer.

\section{Subjects}

The subjects previously had intensive psychoacoustical experience with forced-choice auditory testing $(20-1,000 \mathrm{~h})$. They were either University music majors or had extensive instrumental experience. Each of 346 experimental conditions is represented by the arithmetic mean of 10-19 (median: 16) matchings contributed by six listeners. All listening employed binaural earphones (PRO-4) at about $55 \mathrm{~dB}$ above threshold.

\section{RESULTS}

\section{Effect of Slope and Break Duration}

The magnitude of discrepancy in the visual Poggendorff typically increases as the slopes of the sections increase and as the width of the break increases (Weintraub \& Krantz, 1971, Figure 1). Figure 2 shows corresponding results with the auditory Poggendorff. The abscissa is the duration of the temporal break between the leading and following sections. The unchanging conditions of the tests are shown in the insert of the graph, e.g., in Figure 2, eight steps were employed, each $0.125 \mathrm{sec}$ in duration, with a targeted interpulse interval of $4 \mathrm{msec}$. The change in interpulse interval between successive steps, $s(I)$, is the parameter on the curves.

The matching interpulse interval, $\mathbf{M}$, is expressed in the left panel of Figure 2, relative to $T(\bar{B})$, the targeted interval assuming no break. In these terms, a matching ratio below 1.0 implies a failure to extrapolate the step gradient during the break beyond the simple extrapolation from $J$, the last step before the break. Most of the matches yielded a ratio above 1.0, representing Regions 2 or 3 in Figure 1D.

The matching interpulse interval, $\mathbf{M}$, is expressed in the middle panel of Figure 2 relative to a complete extrapolation, $\mathrm{T}(\mathrm{B})$, during the break. In these terms, a ratio above 1.0 implies the overextrapolation of the step gradient, representing Region 3 of Figure 1D. Most of the matches yield a ratio below 1.0. Coupling the results of the left and middle panels, we can 


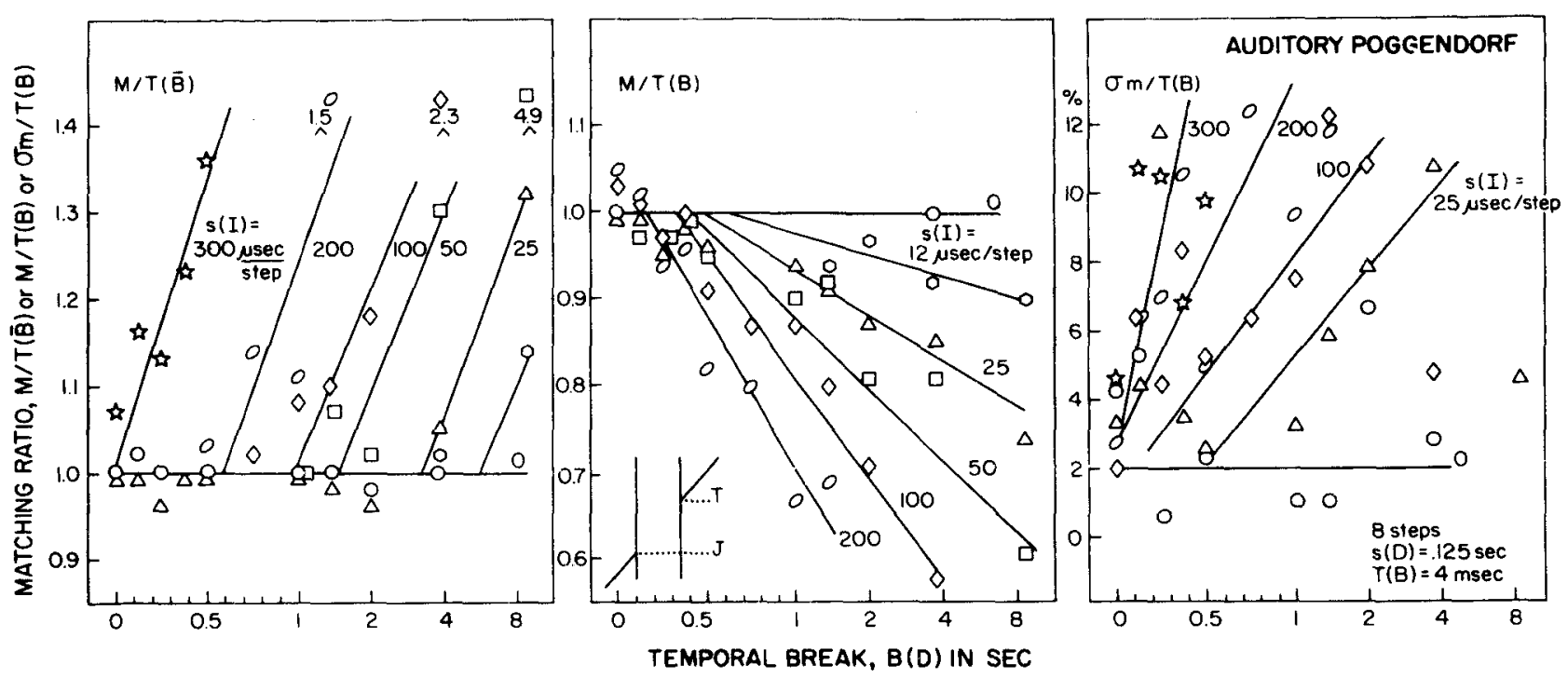

Figure 2. Extrapolation of step tones across a temporal break. The ordinate for the left panel is the matching interpulse interval, $M$, expressed relative to a targeted interpulse interval assuming no temporal break, $T(\bar{B})$. The ordinate for the middle panel is $M$, expressed relative to the targeted interpulse interval assuming the full temporal break, $T(B)$. The ordinate for the right panel is the estimated standard error of the mean, expressed as a percentage of the targeted in terpulse interval. The parameter on all curves is the interval change between successive steps. In Figure 2, and all subsequent graphs, unless specifically noted, all curves are drawn through the data by eye and are without theoretical significance. In all figures, unless specifically noted, the targeted interpulse interval, $T(B)=4 \mathrm{msec}$; the leading and terminal sections consisted of eight steps, $s(N)=8$; and the duration of the temporal break, $B(D)=1.0$ sec.

conclude that the bulk of the matches fell within Region 2, representing an underextrapolation of the step gradient across the break.

In both the left and middle panels of Figure 2, systematic changes are observed with the slope of the interval change, $s(I)$, shown as the parameter on the curves. The magnitude of the departures from a matching ratio of 1.0 is related to the slope of the step gradient, with larger and larger departures obtained at larger and larger slopes.

To a first approximation, the separate curves in the middle panel of Figure 2 project toward a ratio of 1.0 at break $O$, with the slopes of the curves roughly proportional to $s(I)$-the interval change between successive steps. Such a family of curves would be expected if the crucial variable were the total interval change during the break.

The right panel shows the variability of the matches. The variability is also directly related to the interval change between successive steps.

\section{Effect of Step Duration; Number of Steps Constant}

Figure 2 encompasses a wide range of auditory slopes and break durations. As such, the results do not identify the crucial variable, although it was suggested that the crucial variable affecting the discrepancy in the matching was the total interval change during the break.

Figure 3 examines this variable with a wide range of step durations (parameter) with a constant break duration of $1.0 \mathrm{sec}$. A small step duration implies many step changes during the break; a large step duration implies few extrapolated step changes during the break. A matching ratio of 1.0 implies a complete extrapolation of the step gradient; the

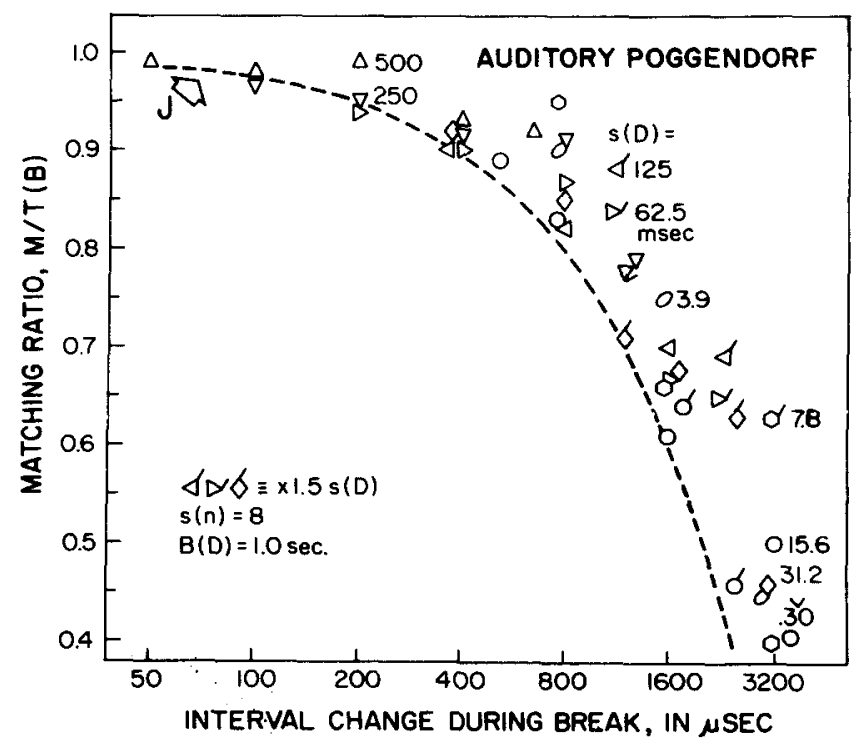

Figure 3. Extrapolation of step tones across a temporal break of $1.0 \mathrm{sec}$ for sequences of eight step durations, $s(D)$, between 3.9 and 500 msec. The dashed line represents no extrapolation beyond the final step tone of the initial segment. In Figures 3 and 4 , the level of the parameter is multiplied by 1.5 for points with a tail. 
lower curve represents the failure of extrapolation over the break. Points intermediate between the dashed curve and an ordinate ratio of 1.0 represent Region 2 or incomplete extrapolations. (The tags appended to the points simply represent step durations of 1.5 times the respective parameter value.)

The main point of Figure 3 is that Region 2 again claims the overwhelming proportion of the data; and, to a first approximation, the matching ratio is determined by the interval change during the break independently of step duration and/or the equivalent number of steps during the break. Closer examination of the points suggests that, for a given interval change during the break, which can be represented by any vertical cut through Figure 3, larger departures are obtained for the shorter step durations. Stated differently, the auditory Poggendorff does not appear to be an artifact of the step changes employed. Indeed, larger and larger discrepancies appear to be obtained with smaller and smaller step durations.

\section{Effect of Step Duration; Section Duration Constant}

Before accepting the previous conclusion, we must attempt to remove an important confounding within the tests of Figure 3. With the fixed number of steps employed in the tests of Figure 3, the duration of the leading or of the following section is directly proportional to step duration. To remove this confounding, the tests of Figure 4 employed a leading and a following section of a constant duration. The product of the number of steps by the step duration was equal to $1.0 \mathrm{sec}$. Such an operation, however, necessarily introduces another confounding: With a leading or following section of a constant duration, $s(I)$ and $s(D)$ must be reciprocally related.

Again, the vast majority of the data fall within Region 2. Otherwise, no strong generalization emerges from Figure 4. Veridical extrapolation, represented by a matching ratio of 1.0 , is approached for a large number of intervals, $S(I) \geqslant 128$, for large interval changes. But these very same conditions yielded the largest deviations from a matching ratio of 1.0 for intermediate interval changes. The inconsistency suggests that we have failed to identify the critical conditions under which the step gradient makes its contribution independently of other features.

\section{Effect of Targeted Interval}

The tests of Figures 2-4 were confined to a targeted interval, $T(B)=4 \mathrm{msec}$. How general are the results for different targeted intervals? Figure 5 represents tests carried out with $\mathrm{T}(\mathrm{B})=0.25,1,4$, and $16 \mathrm{msec}$ with a 0 -sec break and with a 1.0 -sec break. The data may be collapsed across $T(B)$ by expressing $M$ and $s(\mathrm{I})$, each relative to $\mathrm{T}(\mathrm{B})$. There is an overextrapola-

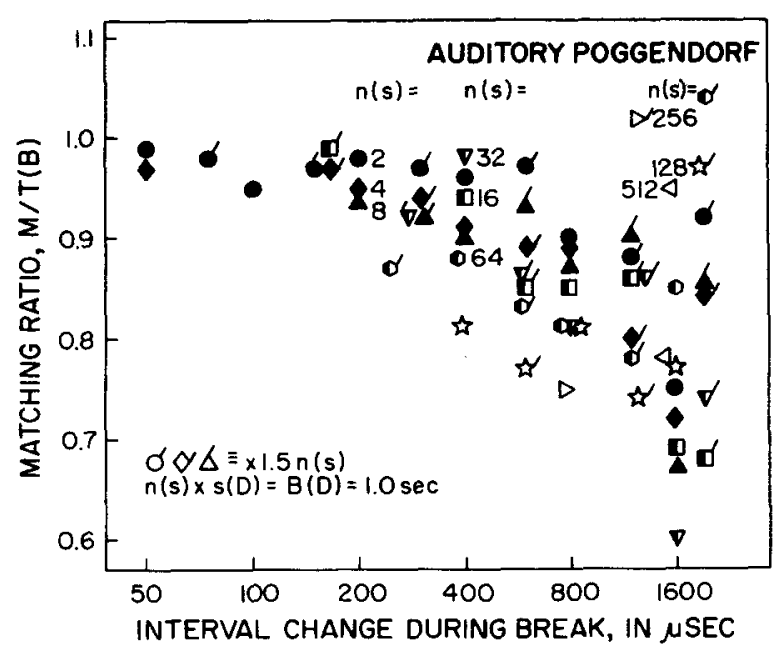

Figure 4. Extrapolation of step tones across a temporal break of $1.0 \mathrm{sec}$ for sequences of $2-512$ steps presented within a 1.0 -sec period. The parameter is the number of steps in the leading, break, and trailing sections. Sequences with a small number of steps are plotted as filled points; sequences with a large number of steps are plotted as open points; and sequences with an intermediate number of steps are plotted as half-filled points.

tion for a 0-sec break, but a consistent underextrapolation for a 1.0-sec break. Thus, we can conclude that the auditory Poggendorff is obtainable over a wide range of auditory frequencies.

\section{Effect of Sound vs. Step Duration}

When successive steps follow immediately upon each other, as illustrated in Figure 1D, there is a necessary interrelation between the duration per step,

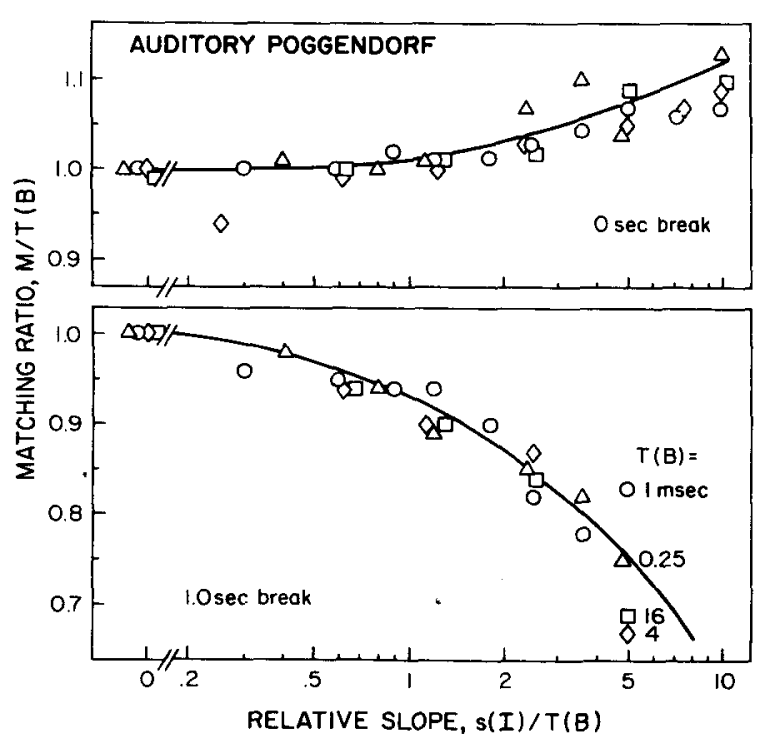

Figure 5. Extrapolation of step tones across a temporal break for a range of targeted frequencies between 0.25 and $4 \mathrm{msec}$. The upper panel presents the matching ratio with a zero temporal break; the lower panel presents the matching ratio with a temporal break of 1 sec. 
the number of steps, and the total duration per section. One way to unconfound these variables, in part, is to introduce a silent interval between successive steps. This permits one to vary the duration of the sound independently of the step duration. This procedure is analogous to the fragmentation of the visual slopes by Weintraub and Krantz (1971).

Figure 6 shows the results of tests with a constant step duration ("on" plus "off" times) of $125 \mathrm{msec}$ and with a constant interval change between successive steps, $\mathrm{s}(\mathrm{I})=200 \mu \mathrm{sec} / \mathrm{step}$. Concretely, an on-time fraction of 0.5 in Figure 6 implies that the signal was "on" for $62.5 \mathrm{msec}$, and "off" for $62.5 \mathrm{msec}$. Note that the number of steps in the break interval-here eight steps in a 1.0 -sec break-is independent of the on-time fraction.

In Figure 6, the degree of underextrapolation over the break is clearly related to the on-time fraction. The maximum departure from a matching ratio of 1.0 tends to be obtained at an on-time fraction of $100 \%$, which was the condition of all of the previous tests. However, the large departures of individual points from the curves again tend to reduce the confidence in which the generalization can be accepted.

\section{Effect of Unequal Slopes}

The tests of Figures 2-6 employed equal slopes for the leading and following sections. Figure 7 examines the effect of modifying the slopes of the separate sections. The inserts describe the constant and variable slopes employed: the left panel represents a zero leading slope and a variable following slope; the middle panel represents a variable leading slope and a zero following slope; the right panel represents a constant leading slope, $s(I)=200 \mu \mathrm{sec} / \mathrm{step}$, and a variable following slope.

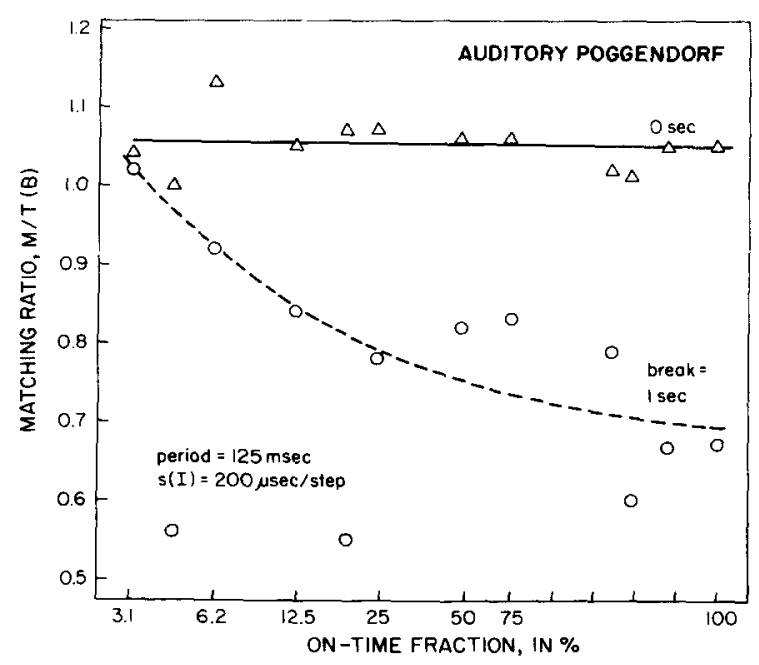

Figure 6. Extrapolation of step tones across a temporal break as a function of the fraction of a step duration of 125 msec that the tone was "on" rather than "off."

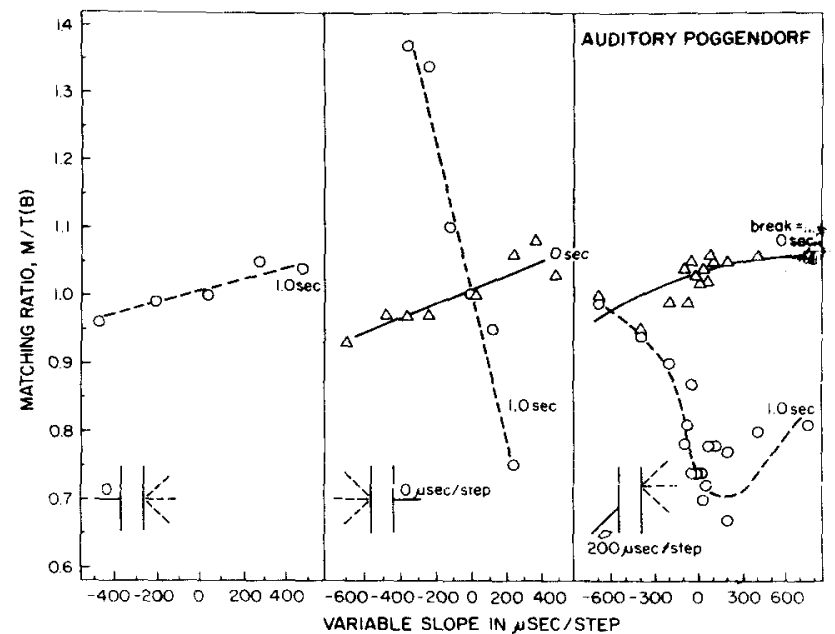

Figure 7. Extrapolation of step tones across a temporal break of $1.0 \mathrm{sec}$ (solid curves) for sequences where the slopes of the leading and following sections are not necessarily equal. The left panel represents conditions with zero leading slope and a variable following slope; the middle panel represents conditions with a zero following slope and a variable leading slope; the right panel represents conditions with a leading slope of $200 \mu \mathrm{sec} / \mathrm{step}$ and a variable following slope.

With a zero leading slope, as shown in the left panel, accurate extrapolation is possible even with a delay of $1.0 \mathrm{sec}$. The next two panels contrast a $1.0-$ sec delay with no delay. In each case, the departure from a matching ratio of 1.0 is greater with the 1.0-sec delay.

The middle panel shows that the magnitude of the departure systematically changes with the direction and slope magnitude of the leading section. Note that underestimations of the targeting interval are represented by matching ratios greater than 1.0 for variable positive slopes. Underestimation is observed for both positive and negative slopes. This result suggests that the auditory Poggendorff is not confined to slopes of one direction.

The right panel shows that slope departures of the following section from the slope of the leading section have an effect related to the direction and to the magnitude of the departure. The minimum matching ratio, or maximum discrepancy, is obtained when the slopes of the leading and terminal sections are equal. By contrast, Weintraub and Krantz (1971, Figure 2) find a minimum discrepancy from veridical extrapolation when the slopes of the two sections are equal.

\section{Logarithmic Steps}

Tests are also carried out with successive steps of the interpulse interval logarithmically spaced, as in the musical scale. We were specifically concerned that the failure to extrapolate accurately was due to the nonmusical linear spacing of successive steps. Fifty-seven experimental conditions, 25-33 matchings 
per condition, were run with eight steps of $125 \mathrm{msec}$ duration leading and following a break of 0 to $1.0 \mathrm{sec}$. The following findings, previously obtained with linear spacing, were confirmed: (1) The magnitude of underestimation increases with the slope ratio (range: 1.00 to 1.15 ) and with the duration of the break interval (range: 0 and $1.0 \mathrm{sec}$ ). (2) The magnitude of the underestimation increases with the on-time fraction of the signal (range: 0.032 to 1.00 ), especially with the 1.0-sec break. (3) With a leading slope ratio of 1.12 and variable following slope ratio, the maximum underestimation was obtained with the following slope ratio at 1.12 .

\section{DISCUSSION}

There is a temptation to draw one analog between auditory time and visual space simply because auditory signals must take place over time while visual signals must take place in space. But, there are also dynamic visual signals which take place over time, so that the correspondence between auditory time and visual space is not mandatory. The analog between visual position and auditory frequency has found expression in several other studies. For example, the paradoxical auditory pitch associated with multioctave tones (Shepard, 1964) is reasonable and understandable when considered in terms of this correspondence, in conjunction with a principal of proximity (Pollack, 1967; Shepard, 1964). Bregman and associates (references in Bregman, 1976, and Dannenbring \& Bregman, 1976) have effectively used the correspondence between visual position and auditory frequency to account for a wide variety of grouping phenomena. Pollack (1975) also employed the same correspondence in an analysis of hidden auditory figures.

But the correspondence between visual position and auditory frequency is not the only useful one. The Cornsweet visual gradient illusion has its audi- tory analog (Jesteadt, Green, \& Wier, 1976) when brightness gradients are reflected in the rise time of auditory sound level. Presumably, if we looked hard enough, a catalog of correspondence might be developed, which in turn would permit more appropriate comparisons of the processing requirements of the auditory and visual systems (Harris, 1950; Julesz \& Hirsh, 1972; Stevens, 1958).

\section{REFERENCE NOTE}

1. Bregman, A. S. The formation of auditory streams. Paper presented at the Seventh International Symposium on Attention and Performance, Marseille, August 1-8, 1976.

\section{REFERENCES}

Dannenbring, G. L., \& Bregman, A. S. Stream segregation and the illusion of overlap. Joumal of Experimental Psychology, Human Perception and Performance, 1976, 2, 544-555.

HARRIs, J. D. Some relations between vision and audition. Springfield, I11: Thomas, 1950.

Jesteadt, W., GreEN, D. M., \& WiER, C. C. Auditory analog to the Craik-O'Brien-Cornsweet illusion in vision. Journal of the Acoustical Society of A merica, 1976, 59, Supplement No. 1, 583.

Julesz, B., \& HIRsh, I. J. Visual and auditory perception-an essay of comparison. In E. E. David \& P. B. Denes (Eds.), Human communication: $A$ unified view. New York: McGrawHill, 1972. Pp. 283-340.

Pollack, I. Selected recent studies of loudness and pitch relevant to sensorineural processes in hearing. In A. D. Graham (Ed.), Sensorineural hearing processes and disorders. Boston: Little Brown, 1967. Pp. 93-103.

Pollack, I. Perception of complex sounds: Hidden auditory figures. Joumal of the Acoustical Society of America, 1975, 58, 1272-1279.

SHEPARD, R. Circularity in judgments of relative pitch. Journal of the Acoustical Society of America, 1964, 36, 2346-2353.

Stevens, S. S. Some similarities between hearing and seeing. The Laryngoscope, 1958, 68, 508-527.

Stevens, S. S. Psychophysics: Introduction to its perceptual, neural, and social prospects. New York: Wiley, 1975.

Weintraub, D., \& KRantz, D. The Pogendorff illusion: Amputations, rotations and other perturbations. Perception \& Psychophysics, 1971, 10, 257-264.

(Received for publication September 24, 1976; revision accepted February 18, 1977.) 